Meta

Journal des traducteurs

Translators' Journal

\title{
Liste des évaluateurs 2012
}

Volume 57, numéro 4, décembre 2012

URI : https://id.erudit.org/iderudit/1021219ar

DOI : https://doi.org/10.7202/1021219ar

Aller au sommaire du numéro

Éditeur(s)

Les Presses de l’Université de Montréal

ISSN

0026-0452 (imprimé)

1492-1421 (numérique)

Découvrir la revue

Citer ce document

(2012). Liste des évaluateurs 2012. Meta, 57(4), 843-846.

https://doi.org/10.7202/1021219ar

Ce document est protégé par la loi sur le droit d'auteur. L'utilisation des services d'Érudit (y compris la reproduction) est assujettie à sa politique d'utilisation que vous pouvez consulter en ligne.

https://apropos.erudit.org/fr/usagers/politique-dutilisation/
Cet article est diffusé et préservé par Érudit.

Érudit est un consortium interuniversitaire sans but lucratif composé de l’Université de Montréal, l'Université Laval et l'Université du Québec à Montréal. Il a pour mission la promotion et la valorisation de la recherche. https://www.erudit.org/fr/ 


\section{Liste des évaluateurs 2012}

Nous remercions chaleureusement tous ceux et celles qui ont donné de leur temps et qui ont contribué à la revue. Toute omission est involontaire de notre part, veuillez nous la signaler.

Sandor Albert (Université de Szeged)

Icíar Alonso-Araguás (Universidad de Salamanca)

Margarita Alonso Ramos (Universidade da Coruña)

Ieda Maria Alves (Universidade de São Paulo)

Mohammad Amouzadeh (University of Isfahan)

Bassey Antia (University of the Western Cape)

Maïte Aragonés Lumeras (World Intellectual Property Organization)

Oslem Albachten (University of the Bosphorus)

Pierre Auger (Université Laval)

Rocío Baños Piñero (Imperial College London)

Josu Barambones Zubiria (Universidad del País Vasco)

Salah Basalamah (Université d'Ottawa)

Nicole Baumgarten (Syddansk Universitet)

Allison Beeby (Universitat Autònoma de Barcelona)

Lieve Behiels (Lessius Antwerpen)

Miguel Bernal-Merino (Roehampton University)

Henri Béjoint (Université Lumière Lyon 2)

Marie-Alice Belle (Université de Montréal)

Sanaa Benmessaoud (Université de Montréal)

Cleci Regina Bevilacqua (Universidade Federal do Rio Grande do Sul)

Esperança Bielsa (Universitat Autònoma de Barcelona)

Xavier Blanco (Universitat Autònoma de Barcelona)

Regina Blass (International University of Africa)

Pier-Pascale Boulanger (Concordia University)

Annie Brisset (Université d'Ottawa)

Joost Buysschaert (Hogeschool Gent)

Caterina Calafat (Universitat de les Illes Balears)

Deborah Cao (Griffith University)

Raymond Chakhachiro (University of Western Sydney)

José Luís Chamosa González (Universidad de León)

Leo Chan (Lingnan University)

Frederic Chaume Varela (Universitat Jaume I)

Ya-mei Chen (National Taipei University of Technology)

Po Suen Maria Cheng (City University of Hong Kong)

Delia Chiaro (Università di Bologna)

Jungwha Choi (Hankuk University of Foreign Studies)

Nicoleta Cinpoes (University of Worcester)

Isabelle Collombat (Université Laval)

Maria da Graça Krieger (Universidade Federal do Rio Grande do Sul)

Marc Delbarge (Lessius University College, Antwerp; College of Europe, Brugges) 
Lucile Desblache (University of Roehampton)

Elena Di Giovanni (Università di Macerata)

Gisele Dionísio da Silva (Universidade Federal de Goiás)

Valérie Dullion (Université de Genève)

Keiran Dunne (Kent State University)

Roch Duval (Université de Montréal)

Domenyk Eades (University of Salford)

Álvaro Echeverri (Université de Montréal)

Maureen Ehrensberger-Dow (Zürcher Fachhochschule)

Sabela Fernández-Silva (Universitat Pompeu Fabra)

Marco Fiola (Ryerson University)

Thierry Fontenelle (Centre de traduction des organes de l'Union européenne)

Antin Fougner Rydning (Universitetet i Oslo)

Clara Foz (Université d'Ottawa)

Ryan Fraser (Université d'Ottawa)

Judit Freixa (Universitat Pompeu Fabra)

Nicolas Froeliger (Université Paris 7 - Diderot)

Chantal Gagnon (Université de Montréal)

Isabel García Izquierdo (Universitat Jaume I)

Joaquín García Palacios (Universidad de Salamanca)

Jeannine Gerbault (Université de Bordeaux)

Anna Ghiglione (Université de Montréal)

Henrik Gottlieb (University of Copenhagen)

Sylviane Granger (Université catholique de Louvain)

Rosalind Greenstein (Université Paris 1 - Panthéon-Sorbonne)

Mathieu Guidère (Université de Toulouse II - Le Mirail)

Camino Gutiérrez Lanza (Universidad de León)

Marie-Noëlle Guillot (University of East Anglia)

Jarjoura Hardane (Université Saint-Joseph de Beyrouth)

Sue-Ann Harding (University of Manchester)

Ghodrat Hassani (University of Kashan)

Anjiang Hu (Sichuan International Studies University)

Claire Huot (University of Calgary)

Amparo Hurtado Albir (Universitat Autònoma de Barcelona)

Moira Inghilleri (University College London)

Matthew Iwuchukwu (University of Nigeria)

Riitta Jääskeläinen (Itä-Suomen yliopisto)

Arnt Lykke Jakobsen (Handelshøjskolen i København)

Laurence Jay-Rayon (Université de Montréal)

Guillaume Jeanmaire (Korea University)

Ronald Jenn (Université Charles-de-Gaulle Lille 3)

Miguel Jiménez-Crespo (Rutgers University)

Ji-Hae Kang (Ajou University)

Thierry Karsenti (Université de Montréal)

Francine Kaufmann (Bar-Ilan University)

Dorothy Kelly (Universidad de Granada)

Georgios Kentrotis (Ionian University)

Don Kiraly (Johannes-Gutenberg-Universität Mainz)

Leong Ko (The University of Queensland) 
Jan-Louis Kruger (North-West University)

Natalie Kübler (Université Paris Diderot - Paris 7)

Francisco Lafarga (Universitat de Barcelona)

Gillian Lane-Mercier (McGill University)

Guy Lapointe (Université de Montréal)

Christopher Larkosh (University of Massachusetts Darthmouth)

Élisabeth Lavault-Olléon (Université Stendhal - Grenoble 3)

Élisabeth Le (University of Alberta)

Claire Le Brun-Gouanvic (Concordia University)

Mu Lei (Guangdong University of Foreign Studies)

Defeng Li (University of London)

Rachel Lung (Lingnan University)

Kazem Lotfipour-Saedi (Université d'Ottawa)

Pedro Macizo (Universidad de Granada)

Brigid Maher (La Trobe University)

Elizabeth Marshman (Université d'Ottawa)

José Luis Martí-Ferriol (Universitat Jaume I)

Rosario Martín (Universidad de Salamanca)

Juan José Martínez Sierra (Universidad de Valencia)

Françoise Massardier-Kenney (Kent State University)

Anna Matamala (Universitat Autònoma de Barcelona)

Roberto Mayoral Asensio (Universidad de Granada)

Iwona Mazur (Adam Mickiewicz University)

Denise Merkle (Université de Moncton)

Reine Meylaerts (Katholieke Universiteit Leuven)

Jasmina Milićević (Dalhousie University)

Livia Monnet (Université de Montréal)

Vicent Montalt i Resurrecció (Universitat Jaume I)

Esther Monzó Nebot (Universitat Jaume I)

Brian Mossop (York University)

Robert Neather (Hong Kong Baptist University)

Josélia Neves (Instituto Politécnico de Leiria)

Christiane Nord (University of the Free State)

Alexis Nouss (Cardiff University)

Sharon O'Brien (Dublin City University)

Eithne O’Connell (Dublin City University)

Minako O’Hagan (Dublin City University)

Riitta Oittinen (University of Tampere)

Pilar Orero (Universitat Autónoma de Barcelona)

Emer O'Sullivan (Leuphana Universität Lüneburg)

Anastasia Parianou (Ionan University)

Daniela Paoleri (Universidad de Granada)

Ana María Pereira (Universidade de Vigo)

Gaëlle Planchenault (Simon Fraser University)

Franz Pöchhacker (Universität Wien)

Sonja Pöllabauer (Karl-Franzens-Universität Graz)

Encarnación Postigo Pinazo (Universidad de Málaga)

Rosa Rabadán (Universidad de León)

Isabelle Rabut (Institut national des langues et civilisations orientales) 
Mariana Raffo (Université de Montréal)

Patrícia Ramos-Reuillard (Universidade Federal do Rio Grande do Sul)

Aline Rémaël (Artesis Hogeschool)

Roda Roberts (Université d'Ottawa)

Margaret Rogers (University of Surrey)

Pablo Romero Fresco (Roehampton University)

Daniel Roush (Eastern Kentucky University)

Rosario Ruan (Universidad de Salamanca)

Myriam Salama-Carr (University of Salford)

Gabriela Saldanha (University of Birmingham)

Vera Lucia Santiago Araújo (Universidade Estadual do Ceará)

Federica Scarpa (Università degli Studi di Trieste)

Christina Schäffner (Aston University)

Anne Schjoldager (Handelshøjskolen i Århus)

Adriana Şerban (Université Paul-Valéry Montpellier 3)

Gregory Shreve (Kent State University)

Maria Sidiropoulou (National and Kapodistrian University of Athens)

Sherry Simon (Concordia University)

Paola Smecca (Università di Catania)

Gérard Snow (Université de Moncton)

Jun Tang (Southeast University)

Christopher Taylor (Università degli Studi di Trieste)

Mohammad Ahmad Thawabteh (Al-Quds University)

Sonja Tirkkonen-Condit (University of Eastern Finland)

Kumiko Torikai (Rikkyo University)

Monica Turci (Università di Bologna)

Roberto Valdeón (Universidad de Oviedo)

Cristina Valderrey (Universidad de Salamanca)

Gudrun Vanderbauwhede (Katholieke Universiteit Leuven)

Gert Vercauteren (Artesis Hogeschool)

Luise von Flotow (Université d'Ottawa)

Baorong Wang (Zhejiang University of Finance and Economics)

Kelly Washbourne (Kent State University)

Malcolm Williams (Université d'Ottawa)

Michaela Wolf (Karl-Franzens-Universität Graz)

Dorothy Wong (Lignan University)

Elizabeth Woodward (Universidad de La Coruña)

Zhijie Wu (Nanjing University of Science and Technology)

Patrick Zabalbeascoa (Universitat Pompeu Fabra)

Soledad Zárate (Imperial College London)

Palma Zlateva (University of Sofia)

Chunshen Zhu (City University of Hong Kong) 